\title{
Two cases of neuronopathic form of Gaucher disease - diagnostic difficulties
}

\author{
Grazina Kleinotiene' ${ }^{1}$ A usteja Ivaskeviciene ${ }^{1,2}$ and Anna Tylki-Szymanska ${ }^{3}$ \\ 'Vilnius University, Faculty of Medicine, Vilnius, Lithuania; ${ }^{2 V i l n i u s ~ U n i v e r s i t y ~ H o s p i t a l ~ S a n t a r o s ~ K l i n i k o s, ~ L i t h u a n i a ; ~}{ }^{3 T h e}$ Children's Memorial Health \\ Institute, Warsaw, Poland
}

Background: Gaucher disease is one of the most common inherited lysosomal storage diseases caused by the deficiency of the enzyme $\beta$-glucocerebrosidase, leading to the accumulation of glucocerebroside. Depending on the clinical manifestations, two different forms of the disease are distinguished - the non-neuronopathic form (type 1) with a variety of presentations - from asymptomatic to symptomatic patients (characterized by hepatosplenomegaly, thrombocytopenia, anemia and osteopenia), and the neuronopathic form (known as types 2 and 3). Besides visceral, osseous, and hematopoietic organ lesions, neuronopathic forms are associated with central nervous system involvement (bulbar and pyramidal signs, horizontal saccadic eye movements, myoclonic epilepsy, progressive development delay). In type 2, the neurological symptoms appear earlier and are more severe, the survival time is shorter. In type 3, the neurological symptoms are milder and allow patients to live a fully productive life. Case presentation: This article includes a review of two cases of neuronopathic Gaucher disease: type 2 and severe type 3. Both patients presented symptoms during infancy and the manifestations were similar but varied in intensity and the dynamics of progress. Enzyme replacement therapy was started in both cases, which decreased visceral symptoms. Conclusions: Both described cases indicate the lack of knowledge and the tendency of doctors to disregard the possibility of Gaucher disease in their paediatrics patients.

Keywords: Gaucher disease, neuronopathic forms lysosomal storage disease, enzyme replacement therapy, opisthotonus, bulbar and pyramidal signs

Received: 30 June, 2021; revised: 09 August, 2021; accepted:

11 August, 2021; available on-line: 14 December, 2021

凶e-mail: grazina.kleinotiene@santa.It

Abbreviations: ALT, alanine aminotransferase; AST, aspartate aminotransferase; BCG, Bacillus Calmette-Guérin; Chito, chitotriosidase; CNS, central nervous system; ERT, enzyme replacement therapy; F, female; GD, Gaucher disease; Hgb, haemoglobin; LysoGb1, glucosylsphingosine; $\beta$-glu, $\beta$-glucocerebrosidase

\section{BACKGROUND}

Gaucher disease (GD) is the most common lysosomal storage disorder caused by the deficiency of acid $\beta$-glucocerebrosidase activity, resulting in the accumulation of glucocerebroside. GD is classified into two types: non-neuronopathic form (type 1) and neuronopathic forms - type 2 GD (acute neuronopathic form) and type 3 GD (chronic neuronopathic form) (Stirnemann et al., 2017).

Distinguishing GD 2 from GD 3 before 2 years of age can be challenging in some cases. It is believed that the classification into types 2 and 3 is unjustified, because these phenotypes are on a continuum. The symptoms occurring in childhood are similar and differ mainly in severity, progression rate and the age of onset. However, the classification into types 2 and 3 is useful from the clinical perspective (Goker-Alpan et al., 2003).

Infantile type $2 \mathrm{GD}$ is characterized by an early onset of rapidly progressive neurological symptoms, including hypotonia/hypertonia, opisthotonus, impaired cognition, hearing and vision, ocular apraxia, strabismus, and bulbar and pyramidal signs (Roshan Lal et al., 2020). The severity and progression of the swallow function may be a prognostic factor for the further course of the disease. Noticeable deterioration in swallowing is a common finding leading not only to cachexia but most often to aspiration pneumonia (Seehra et al., 2019). Type 3 GD varies greatly in terms of the complex of neurological symptoms and their severity. Ethnic differences are noted. Homozygosity for the L444P mutation is the most common genotype in patients with type 3 GD (TylkiSzymańska et al., 2010).

The majority of GD2 and, less frequently GD3 patients, develop infiltrative lung disease due to the accumulation of Gaucher cells in the lung parenchyma and, especially in GD2, in the alveolar spaces (lipid pneumonia) (de Farias et al., 2017).

The aim of the study is to present two cases of neuronopathic Gaucher disease in Lithuanian patients and the diagnostic difficulties.

\section{CASES PRESENTATION}

The study presents two case reports of the patients with neuronopathic forms GD, acute and chronic neuronopathic form, respectively. Retrospective chart review of the patients' medical records, including clinical, biochemical, and molecular phenotype were analyzed. The parents of both of patients signed the informed consent to publish forms.

Detailed patients' data is presented in Table 1.

\section{Case 1}

Patient 1 was the first child of Lithuanian parents born from an uncomplicated pregnancy with a birth weight of $3300 \mathrm{~g}$ and length of $50 \mathrm{~cm}$. At the age of 2 months, the skin abscess appeared on the left upper arm in the area of BCG vaccination (Fig. 1). Chest radiography showed changes suggestive of miliary tuberculosis. Therefore, an intensive treatment with 4 antitubercular drugs (isoniazid, rifampicin, ethambutol, pyrazinamide) was initiated. 


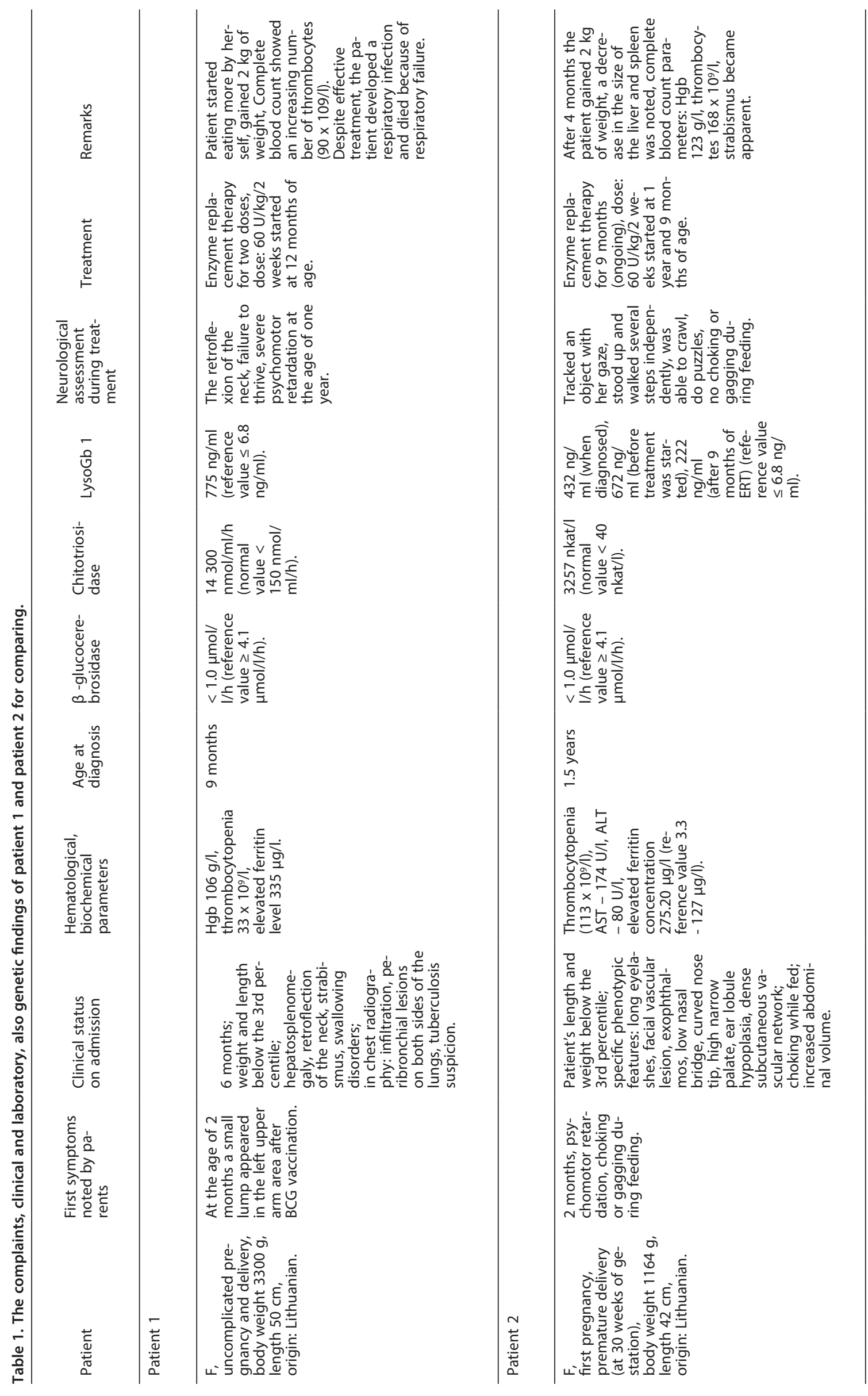




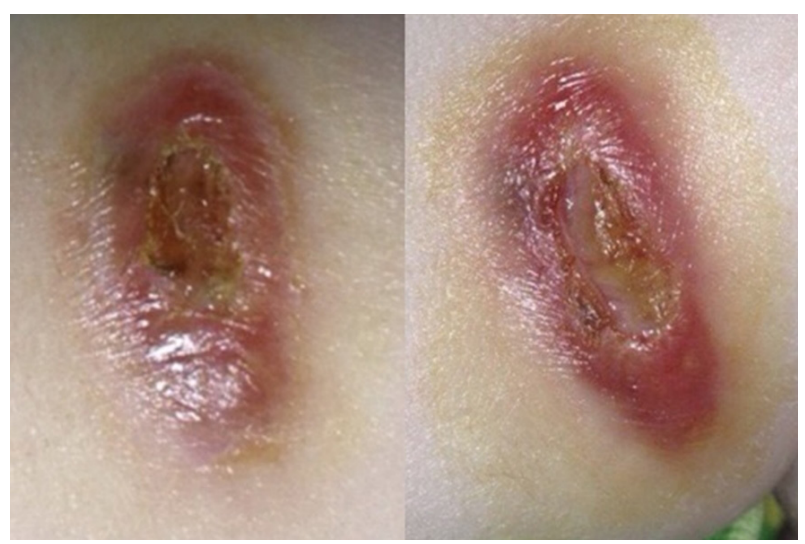

Figure 1. An ulceration in the upper arm at the BCG vaccine injection site.

At the age of 6 months, the infant's weight and length were below the $3 \mathrm{rd}$ percentile. A progressive developmental delay accompanied by the opisthotonus, strabismus, and hepatosplenomegaly were observed.

Laboratory analyses showed the presence of normocytic normochromic anemia and elevated ferritin level (over $100 \mu \mathrm{g} / \mathrm{l}$ ). At the age of 8 months, chest computed tomography (CT) and lumbar puncture (LP) were performed what finally excluded the tuberculosis diagnosis and the treatment with antitubercular drugs was discontinued.

At the age of 9 months, the patient was admitted to the Children's Oncohematology Department with the suspicion of primary immunodeficiency. At admission, the patient's weight and length were below the $3^{\text {rd }}$ percentile. Marked hepatosplenomegaly, opisthotonus and strabismus were still noted (Fig. 2). Chest radiography revealed features of lipid pneumonia (Fig. 3). Laboratory analyses showed the presence of normocytic normochromic anemia (Hgb $106 \mathrm{~g} / \mathrm{l})$, thrombocytopenia (33×109/1), and significantly elevated ferritin level $(335 \mu \mathrm{g} / \mathrm{l})$.

Based on those clinical and biochemical features, Gaucher disease was suspected. The results were as following: decreased activity of leukocyte $\beta$-glucocerebrosidase $(<1.0 \mu \mathrm{mol} / \mathrm{l} / \mathrm{h}$ (reference $\geq 4.1 \mu \mathrm{mol} / \mathrm{l} / \mathrm{h})$ ), increased activity of chitotriosidase $(14300 \mathrm{nmol} / \mathrm{ml} / \mathrm{h}$ (reference $10-150 \mathrm{nmol} / \mathrm{ml} / \mathrm{h})$ ), increased level of lysoGb1 (775 $\mathrm{ng} / \mathrm{ml}$ (reference $\leq 6.8 \mathrm{ng} / \mathrm{ml}$ )). The patient was found to be a compound heterozygote for two known

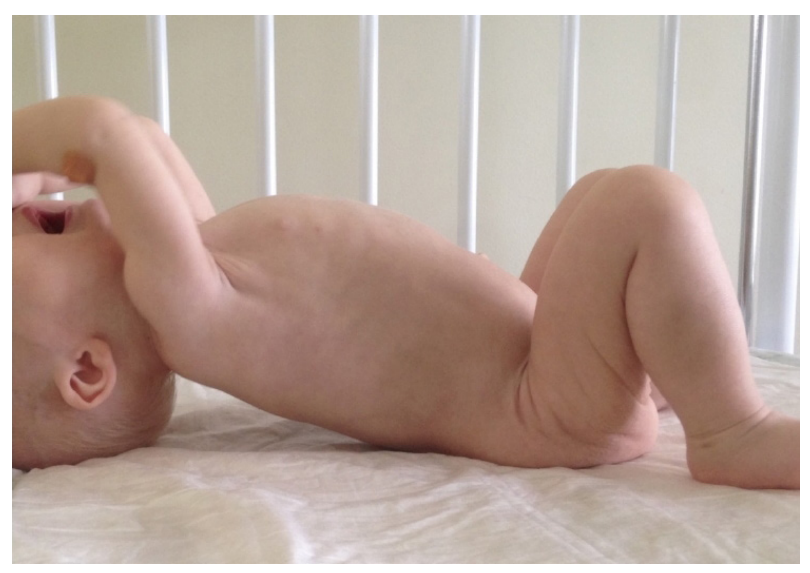

Figure 2. Examination revealed a characteristic retroflexion of the neck. pathogenic variants in the $G B A 1$ gene: c.604C $>\mathrm{T}$, (p.Arg202Ter), and c.1448T $>$ C, (p.Leu483Pro). Both parents were found to be the carriers of those variants. The diagnosis of Gaucher disease, acute neuronopathic form (type 2 GD), was confirmed. Thus, enzyme replacement therapy (ERT) (60 U/kg) was started. After the 2 nd dose the patient started eating more, and liver and spleen volume decreased. The complete blood count showed an increasing number of thrombocytes $\left(90 \times 10^{9} / 1\right)$. However, neurological symptoms, including opisthotonus, severe psychomotor retardation, and failure to thrive progressed. At the age of one year, the patient developed a respiratory infection and died because of respiratory failure.

\section{Case 2}

Patient 2 was born from the 1 st pregnancy and delivered prematurely at 30 weeks of gestation with a birth weight of $1164 \mathrm{~g}$. On prenatal ultrasound examination, foetal growth and weight retardation were observed. At 3 months of age, the patient developed difficulty swallowing. At the age of 9 months, an increased muscle tension was noted. The patient's psychomotor development was delayed: at the age of 1 year, the patient did not sit independently, stand or crawl. Enlarged abdomen, with liver and spleen enlargement and decreased platelet count were found.

At 1.5 years of age, the patient was admitted to the Pediatric Oncohematology Department due to a mild thrombocytopenia. The length $(69 \mathrm{~cm})$ and body weight $(6.9 \mathrm{~kg})$ were below the 3 rd percentile. Phenotypically, some dysmorphic features were noted, such as long eyelashes, a facial vascular lesion, exophthalmos, low nasal bridge, curved nose tip, high narrow palate, ear lobule hypoplasia, rich subcutaneous vascular network. Opisthotonus and occasional strabismus were observed. The muscle tone was significantly increased in the arms and legs. The patient choked while eating.

Abdominal ultrasound revealed a significant enlargement of the liver $(85 \mathrm{~mm})$ and spleen $(103 \mathrm{~mm})$. Laboratory analyses showed the presence of thrombocytopenia $\left(113 \times 10^{9} / 1\right)$, slightly increased liver enzymes (AST $174 \mathrm{U} / 1$, ALT $80 \mathrm{U} / \mathrm{l}$ ), and elevated ferritin level $275.20 \mu \mathrm{g} / 1$ (reference value 3.3-127 $\mu \mathrm{g} / \mathrm{l}$ ).

Based on those clinical and biochemical features, Gaucher disease was suspected. The results were as following: decreased activity of $\beta$-glucocerebrosidase $<1 \mu \mathrm{mol} / \mathrm{l} / \mathrm{h}$, (reference value $\geq 4.1 \mu \mathrm{mol} / \mathrm{l} / \mathrm{h}$ ), elevated level of lysoGb1 $432 \mathrm{ng} / \mathrm{ml}$ (reference value <6.8 ng/

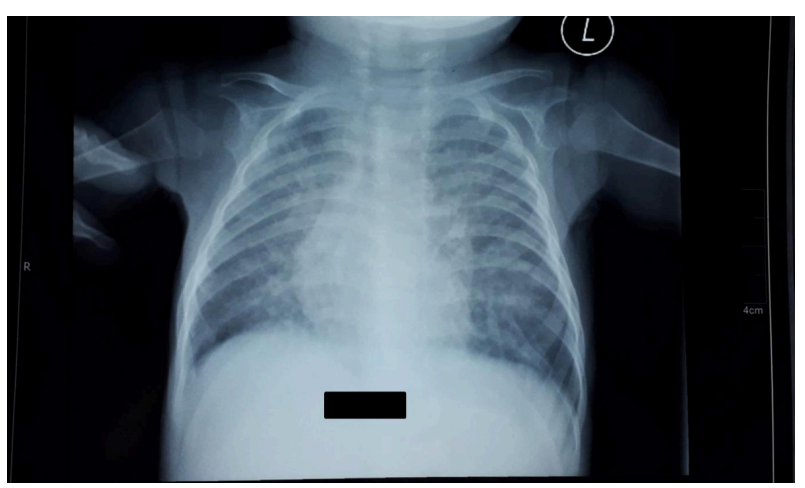

Figure 3. The chest radiography.

There are bilateral small diffuse interstitial nodules with pulmonary oedema component, described as lipid pneumonia by the radiologist. 
$\mathrm{ml}$ ), elevated chitotriosidase level 3257 nkat/1 (normal value $<40$ nkat/l).

Two heterozygous pathogenic variants in the GBA1 gene were identified: c. 604C $>$ T, p. (Arg202Ter) and c. $1342 \mathrm{G}>\mathrm{C}$, (p.Asp448His). ERT was started at 1 year and 9 months of age involving the administration of Cerezyme $^{\circledR}$ (imiglucerase) $60 \mathrm{U} / \mathrm{kg}$ every 2 weeks.

ERT had a positive effect on the general condition of the patient. After 4 months of ERT, the volume of the liver and spleen normalized. Hematological parameters improved. Complete blood count revealed the normal count of thrombocytes $\left(168 \times 10^{9} / 1\right)$. Strabismus became apparent.

After 9 months of ERT, lysoGb1 decreased significantly (222 ng/ml (reference value $<6.8 \mathrm{ng} / \mathrm{ml})$ ).

The patient tracked an object with her gaze, took interest in colourful videos, reached for toys, and maintained a sitting position independently. She could stand and walk several steps independently. During the observation period, the neurological symptoms remained stable. The improvement of the somatic condition due to the ERT contributed to the better general functioning and increased the developmental progress. The parents reported no choking or gagging during feeding. The muscle tone of the upper limbs was variable with a considerable hypertonia of the lower limbs without asymmetry. Tendon reflexes were symmetrically reduced, no pathological reflexes were noted. The Gross Motor Function Classification System assessment revealed level II/III.

\section{DISCUSSION AND CONCLUSIONS}

The study presented two different phenotypes of neuronopathic Gaucher disease (both neurological and visceral) in terms of the severity of symptoms, the progression rate and general disease outcome.

Patient 1 showed a rapid neurological deterioration. However, the patient was initially misdiagnosed with tuberculosis due to the presence of lung lesions which were found characteristic of type 2 GD. Patient 2 presented less severe and later-onset neurological symptoms. In this case, prematurity distracted the doctors from linking the symptoms to GD.

The result of the molecular examination showed in the case of patient 2 a genotype found in patients with chronic neuronopathic GD (formerly type 3) (c. 604C $>$ T, p. (Arg202Ter) and c. 1342G $>$ C, (p.Asp448His)). In this patient, the symptoms were less severe and appeared a little later. So, the prognosis in this case may be more successful as it is in chronic neuronopathic GD.

Comparing the course of the disease in both patients, there is a difference in the severity and the onset age of symptoms. However, Gaucher disease was not considered by doctors in both cases.

Both described cases indicate the lack of knowledge and the tendency of doctors to disregard the possibility of a rare disease in their patients.

The introduction of ERT improved the patient's outcome, regarding visceral symptoms and haematological parameters. In Patient 1, despite the lack of improvement on the CNS, the improvement of the general condition justified ERT. Improving the overall health of the child also allows improving the natural development trend. However, this should not be understood as having a therapeutic effect on the CNS.

\section{Ethics approval and consent to participate}

The parents of both of patients signed the informed consent to publish forms.

\section{Consent for publication}

The parents of both of patients signed the informed consent to publish forms.

\section{Availability of data and material}

Retrospective chart review of the patients' medical records, including clinical, biochemical, and molecular phenotype were analysed.

\section{Competing interests}

The authors have no conflicts of interest to declare.

\section{Authors' contributions}

All authors discussed the final version and contributed to the manuscript.

\section{Acknowledgements}

The authors would like to thank the patients and their families for their consent to participate in this study and to use their data in this publication.

\section{REFERENCES}

de Farias LPG, Padilha IG, Dos Santos CJJ, Maranhão CPM, de Miranda CMNR (2017) Pulmonary involvement in Gaucher disease. Radiol Bras 50: 408-409. https://doi.org/10.1590/01003984.2016.0081

Goker-Alpan O, Schiffmann R, Park JK, Stubblefield BK, Tayebi N, Sidransky E (2003) Phenotypic continuum in neuronopathic Gaucher disease: an intermediate phenotype between type 2 and type 3. J Pediatr 143: 273-276. https://doi.org/10.1067/S00223476(03)00302-0. PMID: 12970647.

Roshan Lal T, Seehra GK, Steward AM, Poffenberger CN, Ryan E, Tayebi N, Lopez G, Sidransky E (2020) The natural history of type 2 Gaucher disease in the 21 st century: A retrospective study. Neurology 95: e2119-e2130. https://doi.org/10.1212/ WNL.0000000000010605

Seehra G, Solomon B, Ryan E, Steward AM, Roshan Lal T, Tanima Y, Lopez G, Sidransky E (2019) Five-parameter evaluation of dysphagia: A novel prognostic scale for assessing neurological decline in Gaucher disease type 2. Mol Genet Metab 127: 191-199. https://doi. org/10.1016/j.ymgme.2019.06.002

Stirnemann J, Belmatoug N, Camou F, Serratrice C, Froissart R, Caillaud C, Levade T, Astudillo L, Serratrice J, Brassier A, Rose C, Billette de Villemeur T, Berger MG (2017) A review of gaucher disease pathophysiology, clinical presentation and treatments. Int J Mol Sci 18: 441. https://doi.org/10.3390/ijms18020441

Tylki-Szymańska A, Vellodi A, El-Beshlawy A, Cole JA, Kolodny E (2010) Neuronopathic Gaucher disease: demographic and clinical features of 131 patients enrolled in the International Collaborative Gaucher Group Neurological Outcomes Subregistry. I Inherit Metab Dis 33: 339-346. https://doi.org/10.1007/s10545-009-9009-6 